\title{
Pulmonary Koch in 4 Months Old: Congenital or Acquired?
}

\author{
Priyasha Tripathi ${ }^{1}$, Surendra Singh Raghuwanshi ${ }^{2}$ \\ ${ }^{1,2}$ Senior Resident, Department of Paediatrics, Atalbihari Vajpayee Govt Medical College Vidisha (M.P.) \\ Corresponding Author: Priyasha Tripathi
}

\begin{abstract}
4 months old female child presented to us with complaints of fever, cough, cold, and poor weight gain. On examination the child had failure to thrive (weight $2.75 \mathrm{~kg}$, length $52 \mathrm{cms}$, head circumference $35 \mathrm{cms}$, weight for length <-3SD), cachexic look, severe respiratory distress (bilateral chest retractions and nasal flaring), hepatosplenomegaly, delayed milestones with low birth weight $(2.07 \mathrm{~kg})$, continuous low grade fever. Mother was an open case of pulmonary Koch, taking antitubercular treatment since 6 months. Investigations revealed anemia, neutrophilia with reactive CRP, raised transaminases, pulmonary fibrosis and cavitary lesions in chest x-ray with normal CSF examination. Mantoux was reactive with gastric aspirate and cerebrospinal fluid negative for TB bacilli in GeneXpert. So the question arises, is it congenital or acquired?
\end{abstract}

Key words: congenital TB, hepatosplenomegaly, respiratory distress, failure to thrive

\section{INTRODUCTION}

TB remains a leading cause of morbidity and mortality in developing countries. Congenital TB is fatal if untreated, and morbidity is increased if the diagnosis and treatment is delayed ${ }^{[1]}$. A high suspicion and good screening of mothers and neonates is recommended, clinician should always be aware of the unusual presentation of congenital tuberculosis [1]. BCG vaccine is recommended in all non-high risk neonates if asymptomatic and immunocompetent. Neonate diagnosed with congenital TB should be treated with anti-tubercular drug regimen ${ }^{[2]}$.

Transmission of disease is well known via transplacental transmission through the umbilical vein to the fetus, or through the ingestion of infected amniotic fluid. Early diagnosis of congenital TB is challenging because of its non-specific presentation, it should be distinguished from the more frequent acquired neonatal $\mathrm{TB}$, in which the infant is infected after birth by a person suffering from the disease ${ }^{[2]}$. Only 300 cases were reported till 1989, 58 cases in 1994, from 2001 to December 2005, 18 more cases have been mentioned but only about 10 cases have been reported in India [3]. Congenital TB is rare because tuberculosis primarily causes infertility and also placenta acts as a physical barrier for the bacilli ${ }^{[4]}$. After crossing the placenta through the umbilical vein, a primary focus develops in the fetal liver with involvement of the periportal lymph nodes and secondarily the lungs are infected. The bacilli remain inactive in the fetal lung and after birth when there is an increase in the oxygenation and pulmonary perfusion; the bacilli become active and causes disease. Dissemination occurs via fetal circulation to other organ systems. Clinical manifestation include poor feeding, failure to thrive, lethargy, irritability, cough, respiratory distress, fever, poor feeding, seizure, hepatosplenomegaly and lymphadenopathy. 
Some may present with progressive liver dysfunction without any respiratory symptoms ${ }^{[5]}$.

\section{CASE REPORT}

4 months old female child was admitted in PICU with severe respiratory distress and poor feeding. The child was cachexic, severe failure to thrive with weight $2.75 \mathrm{~kg}$, length $52 \mathrm{cms}$, head circumference $35 \mathrm{cms}$, weight for length <3SD. The child was delivered low birth weight, $2.0 \mathrm{~kg}$ to mother who is an open case of pulmonary Koch. She was taking anti-tubercular treatment since 6 months. The child on examination had gross developmental delay with microcephaly, hepatomegaly, respiratory distress, pallor and multivitamin deficiencies. She was on mixed feeding (breast feeding with Blood investigations revealed anemia, neutrophilia, thrombocytosis, raised CRP, raised transaminases(SGPT> SGOT). Chest $\mathrm{x}$-ray revealed pulmonary fibrosis and cavitatory lesion. Mantoux was reactive but gastric aspirate for GeneXpert was negative. USG abdomen suggested hepatomegaly with mild ascites. CSF was reported normal. The patient was started AKT under DOTS (half tablet of 3FDC with single tablet of ethambutol daily) along with pyridoxine. She was investigated for HIV, hepatitis B and other TORCH group of infections also which were reported negative. IV broad spectrum was given in view of sepsis. Despite all treatment the child kept deteriorating and was shifted to ventilatory support and was ultimately referred to higher center where she succumbed to the disease.

\section{1) 4 months old cachexic female child with hepatomegaly and respiratory distress}

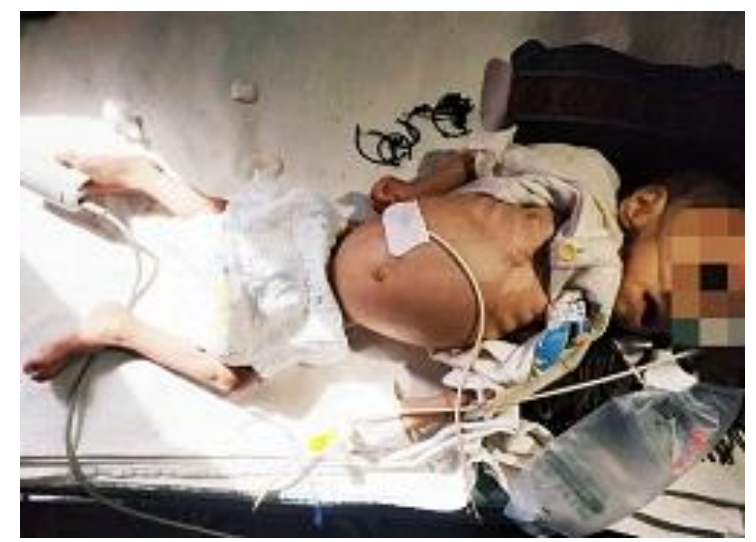

2) Chest x-ray showing pulmonary fibrosis and cavitary lesions

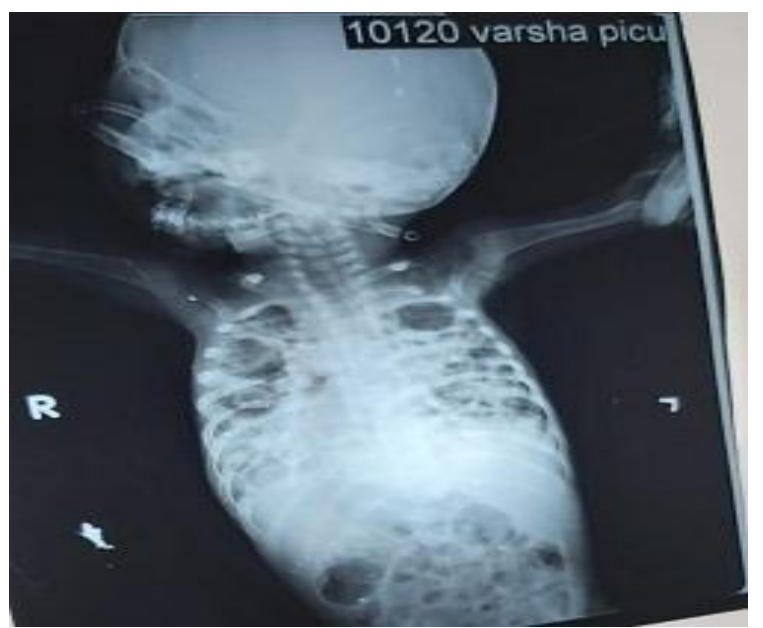

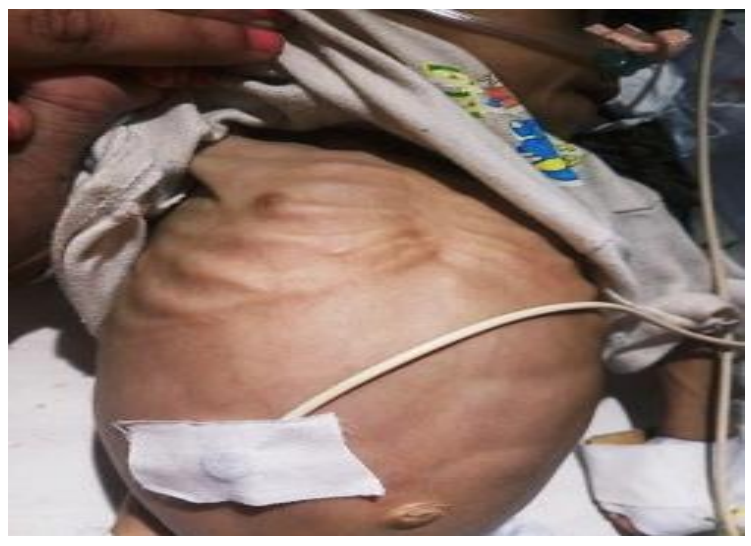

DISCUSSION

Work up of the mother if high suspicion present, should be done including a histological examination of placenta at birth, Mantoux test, chest X-ray and endometrial aspiration and curettage. Once the mother and the neonate are diagnosed with TB, both should undergo screening for Human Immunodeficiency Virus (HIV). Diagnostic criteria for the diagnosis of congenital tuberculosis were first described by Beitzki6 in 1935 and were revised by Cantwell in $1994^{[6]}$.

Revised criteria by Cantwell: Proven tuberculosis lesions in the infant plus one of 
the following: Lesions occurring in the neonate in the first week of life.

- Primary hepatic complex.

- Identification of Mycobacterium tuberculosis in maternal genital tract or placenta.

The demonstration of a hepatic primary complex usually requires an open surgical procedure or autopsy to confirm liver involvement, although the sensitivity of liver biopsy for the diagnosis of congenital tuberculosis is $100 \%{ }^{[7]}$.

\section{AIIMS Protocol 2019:}

One important criterion is the exclusion of postnatal acquisition by thoroughly investigating the close contacts. Congenital TB is diagnosed by the primary criteria along with at least one of the secondary criteria. Prophylaxis is continued for 6 months and after that, if Mantoux negative, isoniazid is stopped. Mother should breastfeed the baby, isolation is recommended only in cases of MDR TB.

Steroid may be used in tubercular meningitis, pericarditis, bronchoalveolar $\mathrm{TB}$, bronchial and mediastinal compression syndrome, and TB IRIS. Prednisolone 1$2 \mathrm{mg} / \mathrm{kg} /$ day or dexamethasone $0.6 \mathrm{mg} / \mathrm{kg} /$ day or its equivalent is given for 4 weeks and then tapered over the next 4 weeks.

AAP and AIIMS recommend that infants receiving prophylaxis should have clinical surveillance every 4-6 weeks and then after completion of treatment follow up should be done every 6 months for 2 years. Compare the weight from the last visit every time during follow up. DOTS recommend chest X-ray at the end of treatment and liver function tests if hepatotoxicity developed.

Congenital TB has a very high mortality rate upto $50 \%$ if presented before 4weeks ${ }^{[3]}$. It is still challenging to distinguish congenital from postnatally acquired tuberculosis. The age at the onset of congenital tuberculosis is not uniform ${ }^{[8]}$. Congenital tuberculosis should be diagnosed if tuberculosis in the infant originated from the mother before birth or at birth.
Symptoms of congenital tuberculosis mainly occur within 3 weeks after birth, at an average age of 28 days, although some patients may not develop symptoms until 3 months of age ${ }^{[9] ~[10]}$. The immune system of neonates is not well developed, and no allergic reactions occur within 2 to 10 weeks after the latest infection, the PPD test can be negative even in patients with severe tuberculosis and the disease cannot be ruled out ${ }^{[11]}$. Most children with congenital tuberculosis have abnormal chest X-ray images, Ultrasonography is also very useful in the diagnosis of liver, spleen and kidney lesions. In countries like India, with high tuberculosis load, the prevalence of active tuberculosis among pregnant women and postpartum women is as high as 60 cases/100,000 population per year, making routine screening for tuberculosis mandatory $[12][13][14]$.

Patients with congenital and acquired tuberculosis should be treated similarly. Infants should receive isoniazid (10-15 mg/kg.d), rifampin (10-20 mg/kg.d), pyrazinamide (15-30 mg/kg.d), and either streptomycin $\quad(20-30 \quad \mathrm{mg} / \mathrm{kg} . \mathrm{d})$ or ethambutol (15-25 mg/kg.d) for the first 2 months, followed by isoniazid and rifampin for 4-10 months, as per the new revised RNTCP guidelines ${ }^{[15]}$.

With so many national programmes and policies attributed exclusively towards preventing and treating tuberculosis at every level, India still lags far behind in proper screening for congenital tuberculosis and it is still very difficult to distinguish between acquired or congenital TB.

Acknowledgement: None

Conflict of Interest: None

Source of Funding: None 


\section{REFERENCES}

1. Kumari $\mathbf{N}$ etal.Congenital Tuberculosis: A review article. Int J ContempPediatr. 2019 Nov; 6(6):2750-2754.

2. Balasubramanian S, Shivram R, Padmasani LN, Nagaraju. Congenital TB.Indian J Pediatr. 1999;66:148-50.

3. Hassang G, Qureshi W, Kadri SM. Congenital tuberculosis. JK Sci. 2006;8:193-4.

4. K Ghosh, K Ghosh, JR Chowdhury. Tuberculosis and female reproductive health.J Postgradmedi. 2011; 55(4):307-13.

5. Parakh A, Saxena R, Thapa R, Sethi GR, Jain S. Perinatal tuberculosis: four cases and use of broncho-alveolar lavage. Ann trop Paediatr. 2011;31:75-80.

6. Chotpitayasunondh T, Sangtawesin V. Congenital tuberculosis. J Med Assoc Thai. 2003;86:689-95.

7. Dewan P, Gomber S, Das S. Congenital tuberculosis: a rare manifestation of a common disease. PaediatrInt Child Health. 2014;34(1):60-2.

8. Li et al. Diagnosis and treatment of congenital tuberculosis: a systematic review of 92 cases. Orphanet Journal of Rare Diseases (2019) 14:131

9. Şen V, Selimoğlu ŞH, Aktar F, Uluca Ü, Karabel M, Fuat GM. Congenital tuberculosis: presentation of a rare case. Arch Argent Pediatr. 2015;113(2):e101-5.
10. Vogel M, Schroten H, Kahl P, Müller A. High latency of tuberculosis manifestation in a premature extremely low birth weight infant with favorable outcome. Neonatology. 2014;105(2):91-4.

11. Obringer E, Heald-Sargent T, Hageman JR. Neonatal tuberculosis. Pediatr Ann. 2015;44(5):e126-30.

12. Mathad JS, Gupta A. Tuberculosis in pregnant and postpartum women: epidemiology, management, and research gaps. Clin Infect Dis. 2012;55(11):1532-49.

13. Aelami MH, Qhodsi RMA, Sasan MS, Ghazvini K. Congenital tuberculosis presenting as ascites. Arch Iran Med. 2011;14(3):209-10.

14. Le DK, Barber N, Doerholt K, Sharland M. Rifampicin pharmacokinetics in extreme prematurity to treat congenital tuberculosis. BMJ Case Rep. 2013;2013.

15. Cantwell MF, Shehab ZM, Costello AM, Sands L, Green WF, Ewing EP, et al. Brief report: congenital tuberculosis. $\mathrm{N}$ Engl $\mathrm{J}$ Med. 1994;330(15):1051-4.

How to cite this article: Tripathi R, Raghuwanshi SS. Pulmonary koch in 4 months old: congenital or acquired? Int $J$ Health Sci Res. 2021; 11(5): 206-209. DOI: https:// doi.org/10.52403/ijhsr.20210532 